\title{
Imbalance of stromelysin- 1 and TIMP-1 in the mucosal lesions of children with inflammatory bowel disease
}

R B Heuschkel, T T MacDonald, G Monteleone, M Bajaj-Elliott, J A Walker Smith, S L F Pender

\begin{abstract}
Background-Degradation of the extracellular matrix and ulceration of the mucosa are major features of inflammatory bowel disease (IBD). One of the most important enzymes in degrading the matrix and produced in excess by cytokine activated stromal cells, is stromelysin-1. The activity of stromelysin- 1 is controlled by tissue inhibitor of metalloproteinase (TIMP-1), its natural inhibitor. In model systems excess stromelysin-1 produces mucosal degradation.

Methods-Quantitative competitive RTPCR was used to analyse stromelysin-1 and TIMP-1 transcripts; western blotting was used to measure the amount of stromelysin-1 and TIMP-1 protein in biopsy samples from children with IBD.

Results-In biopsies from patients with active Crohn's disease $(n=24)$, ulcerative colitis $(n=23)$, and controls $(n=16)$, TIMP-1 transcripts and protein were abundant and unchanged. Stromelysin-1 transcripts and protein were markedly elevated in mucosal biopsies obtained from inflamed sites of patients with active IBD but were not elevated in adjacent endoscopically normal mucosa $(n=10)$. Elevated levels of stromelysin-1 transcripts in active Crohn's disease $(n=5)$ returned to normal levels following treatment with enteral nutrition.

Conclusions-Stromelysin-1 is markedly overexpressed at inflamed sites in patients with IBD whereas TIMP-1 remains unaltered. Excess stromelysin-1 is likely to be responsible for loss of mucosal integrity in IBD.
\end{abstract}

Department of Paediatric

Gastroenterology, Royal Free and University College

Medical School,

London, UK

R B Heuschkel $\dagger$

J A Walker Smith

Department of Paediatric

Gastroenterology, St

Bartholomew's and the

Royal London School

of Medicine and

Dentistry, London, UK

T T MacDonald

G Monteleone

M Bajaj-Elliott

S L F Pender

†Present address:

Combined Program in

Pediatric

Gastroenterology and

Nutrition, Division of

Gastroenterology,

Children's Hospital, 300

Longwood Avenue,

Boston, MA 02115, USA

Correspondence to:

Dr S L F Pender, Division of

Infection, Allergy,

Inflammation and Repair,

Level E, South Academic

Block, Mail point 813,

Southampton General

Hospital, Southampton

SO16 6YD, UK. Email:

s.pender@soton.ac.uk

Accepted for publication

21 December 1999
(Gut 2000;47:57-62)

Keywords: inflammatory bowel disease; enteral nutrition; intestine; matrix metalloproteinase; tissue inhibitor of metalloproteinase

Degradation and remodelling of the extracellular matrix $(\mathrm{ECM})$ is increasingly implicated in the pathogenesis of many inflammatory diseases such as arthritis and inflammatory bowel disease (IBD). ${ }^{1}$ Matrix metalloproteinases (MMPs) play a key role in these events. MMPs can cleave all components of the ECM and in health act in harmony as part of normal tissue turnover. While MMPs are essential in the normal physiology of angiogenesis, uterine ${ }^{2}$ and breast involution, ${ }^{34}$ bone resorption, ${ }^{5}$ and tissue repair, ${ }^{6}$ during inflammation their dysregulation leads to excess degradation of the matrix components and loss of tissue integrity. MMPs are released from almost all connective tissue cells in response to inflammatory stimuli such as cytokines. Most MMPs are secreted as inactive proenzymes and require proteolytic processing of their amino terminal domain to become active. ${ }^{8}$ This may be caused by plasmin, free radicals, or by other proteases. ${ }^{910}$ MMP activity is controlled by their natural inhibitors, tissue inhibitors of metalloproteinases (TIMPs), or by synthetic MMP inhibitors.

MMPs have recently been shown to be important in tissue injury in the gut. We demonstrated that activation of $\mathrm{T}$ cells in explant cultures of fetal human intestine caused mucosal ECM degradation, ${ }^{11}$ mediated by MMPs, in particular stromelysin-1. ${ }^{12}$ The tissue inhibitors of metalloproteinases remained unchanged after stimulation. Recombinant stromelysin-1 caused severe tissue injury when added directly to the explants. In addition, a synthetic inhibitor of MMPs prevented matrix degradation following $\mathrm{T}$ cell activation. Further studies implicating MMPs in IBD came from Saarialho-Kere and colleagues $^{13}$ who used in situ hybridisation to show increased expression of stromelysin-1 in human gastrointestinal ulcers. Bailey et al, using immunofluorescence staining, showed only a slight increase in immunoreactive stromelysin- 1 in areas of smooth muscle proliferation and mucosal damage in patients with Crohn's disease. ${ }^{14}$

In this study we have quantified stromelysin-1 and TIMP-1 transcripts and protein in children with active Crohn's disease and ulcerative colitis (UC), and in normal controls. We analysed levels in endoscopically normal and abnormal mucosa in children with active IBD. We also studied the effect of a course of exclusive enteral nutrition on stromelysin-1 transcripts in the mucosa of children with Crohn's colitis.

\section{Methods}

PATIENTS

Patients underwent video colonoscopy following satisfactory bowel preparation during the

Abbreviations used in this paper: IBD, inflammatory bowel disease; MMPs, matrix metalloproteinase; TIMP-1, tissue inhibitor of metalloproteinase; ECM, extracellular matrix; PCDAI paediatric Crohn's disease activity index; RT-PCR, reverse transcription-polymerase chain reaction; UC, ulcerative colitis. 
previous 24 hours. Parental consent and ethics approval had previously been obtained. $\mathrm{Mu}-$ cosal biopsies were taken from children with active UC and Crohn's disease, and from normal controls (patients suspected of IBD, justifying colonoscopy, but in whom no endoscopic or histological abnormality was detected). Resection tissue was retrieved from several patients following surgery for intractable inflammation. All tissue biopsies were retrieved from sites of macroscopically active inflammation. Biopsies were immediately snap frozen in liquid nitrogen and stored at $-70^{\circ} \mathrm{C}$. Multiple biopsies/specimens were also taken for separate histological assessment. In all cases of acute IBD, histology confirmed the presence of acute inflammatory changes within the colon.

Group $A$

Tissue from 15 patients with Crohn's disease, 14 patients with UC, and seven normal controls was studied. Tissue was obtained from children with clinically active IBD (Crohn's disease, paediatric Crohn's disease activity index (PCDAI) $>30^{15}$; UC, colitis score $>4^{16}$ ). All biopsies and resection tissue were taken from sites of active inflammation within the colon of each child. All patients were receiving aminosalicylate treatment.

Group B

Seven patients with macroscopically active Crohn's disease and three with active UC had paired endoscopically normal/abnormal biopsies taken during colonoscopy. Active mucosal disease was defined as erythema, granularity, loss of vascular pattern, aphthoid ulceration, and occasional frank ulceration. Biopsies were also obtained from endoscopically normal areas as close to the paired abnormal site as possible, but showing none of the above features. Biopsies from the transverse colon were analysed if representative, although the ascending or descending colon was biopsied if dictated by the presence or absence of visible abnormalities. All patients had clinically active disease as defined above.

Group C

Five patients with moderate to severe ileocolonic Crohn's disease (PCDAI mean 37 (range 30-47.5)) were biopsied before and after treatment. Biopsies were always taken from sites of maximal endoscopic inflammation within the colon. If no inflammation was visible, representative biopsies were taken from the mid-transverse colon. All children received an eight week course of exclusive oral polymeric enteral feed (AL110 / CT3211 Nestlé Clinical Nutrition). All patients also received aminosalicylates at standard doses, while two patients required steroids in addition to enteral nutrition, one child being treated acutely with intravenous azathioprine in view of the severity of his Crohn's colitis at presentation. All five children underwent complete clinical (PCDAI 0 (range $0-10)$ ) and histological remission by the time of their repeat endoscopy at eight weeks.
RT-PCR

Competitive quantitative reverse transcriptionpolymerase chain reaction (RT-PCR) was performed for stromelysin-1 and TIMP-1 using synthetic RNA encoding the MMP primer sites (donated by Dr G S Schultz, University of Florida, Florida, USA) according to the method described previously. ${ }^{17}{ }^{18}$ Cytokine stimulated fetal gut mesenchymal cell RNA was used as a positive control for all experiments. $^{12}$

All analyses were performed in a blinded manner, with allocated biopsy numbers only being decoded following quantitative densitometric analysis. This technique allows quantification of the number of MMP transcripts in a tissue sample to 1000 transcripts/ $\mu$ g of total RNA.

WESTERN BLOTTING

Western blotting was performed according to a modified method described previously. ${ }^{12}$ In brief, biopsies were homogenised with lysate buffer (6 M urea and $50 \mathrm{mM}$ Tris- $\mathrm{Cl}$ buffer, $\mathrm{pH}$ 7.6) and the amount of protein was determined by the Bio-Rad Protein Assay (Bio-Rad Laboratories Ltd, Hemel Hempstead, UK). Similar amounts of protein were loaded in each lane and were run on $10 \%$ SDS-PAGE under reducing conditions. After electrophoresis, protein was transferred to nitrocellulose (BioRad); a sheep antihuman stromelysin-1 polyclonal antibody (1/500 dilution, The Binding Site Ltd, Birmingham, UK) and a monoclonal mouse antihuman TIMP-1 antibody $(1 \mu \mathrm{g} / \mathrm{ml}$, Oncogene Research Ltd, Nottingham, UK) were used as primary antibodies. Rabbit antisheep or rabbit-antimouse antibodies conjugated to horseradish peroxidase (1/2500 dilution, Dako Ltd, High Wycombe, UK) were used as secondary antibodies, and the reaction was developed with the ECL plus kit (Amersham Pharmacies, Amersham, UK) or followed by DAB substrate. Computer assisted scanning densitometry (Seescan, Cambridge, UK) was used to analyse the intensity of the immunoreactive bands in the autographs. Where multiple immunoreactive bands were present, the ODs of individual bands were added together to give a measure of total stromelysin-1 in the tissue.

STATISTICAL ANALYSIS

Differences between groups were compared using the non-parametric Mann-Whitney $U$ test.

\section{Results}

MMPS AND TIMP-1 TRANSCRIPTS IN THE ACTIVE LESIONS OF CHILDREN WITH IBD

Stromelysin-1 transcripts were significantly elevated in all patients with active Crohn's disease or active UC compared with normal controls. All normal controls $(n=7)$ had undetectable levels ( $<1000$ transcripts/ $\mu$ g total RNA). Patients with Crohn's disease $(n=15)$ had a median of 100799 transcripts/ $\mu$ g total RNA (range 12 900-809 4505) and patients with UC $(n=14)$ a median of 84803 transcripts/ $\mu \mathrm{g}$ total RNA (range 15 650-399 008). There was 
no significant difference between Crohn's disease and UC $(p=0.33$, Mann-Whitney U test), but in both, stromelysin- 1 transcripts/ $\mu \mathrm{g}$ total RNA were significantly elevated compared with normal controls $(\mathrm{p}<0.00008$ and $\mathrm{p}<0.0002$, respectively, Mann-Whitney U test) (fig 1A).

TIMP-1 transcripts were not significantly different between children with IBD and normal controls (Crohn's disease $(n=16)$ 283382 (range 11 171-1 668 992), UC ( $n=11)$ 98425 (23 059-285 876), normal controls $(\mathrm{n}=5) 102787$ (34 218-971 406)) (fig 1B).

Analysis of TIMP-1/stromelysin-1 transcript ratios showed a significant difference between children with active IBD and normal controls. Children with active UC had a median ratio 100-fold lower than normal controls. Children with active Crohn's disease had a median 17-fold lower than normal controls. Both groups with active IBD differed significantly from normal controls $(p=0.0006, p=0.002$ for Crohn's disease and UC respectively, MannWhitney U test). There was still a significant difference between TIMP-1/stromelysin-1 ratios of children with active Crohn's disease and active UC ( $p=0.056$, Mann-Whitney $U$ test $)$ (fig 1C). We also used the same method to detect two other MMPs, interstitial collagenase and gelatinase $\mathrm{B}$, but the number of transcripts were often less than 1000 and showed no
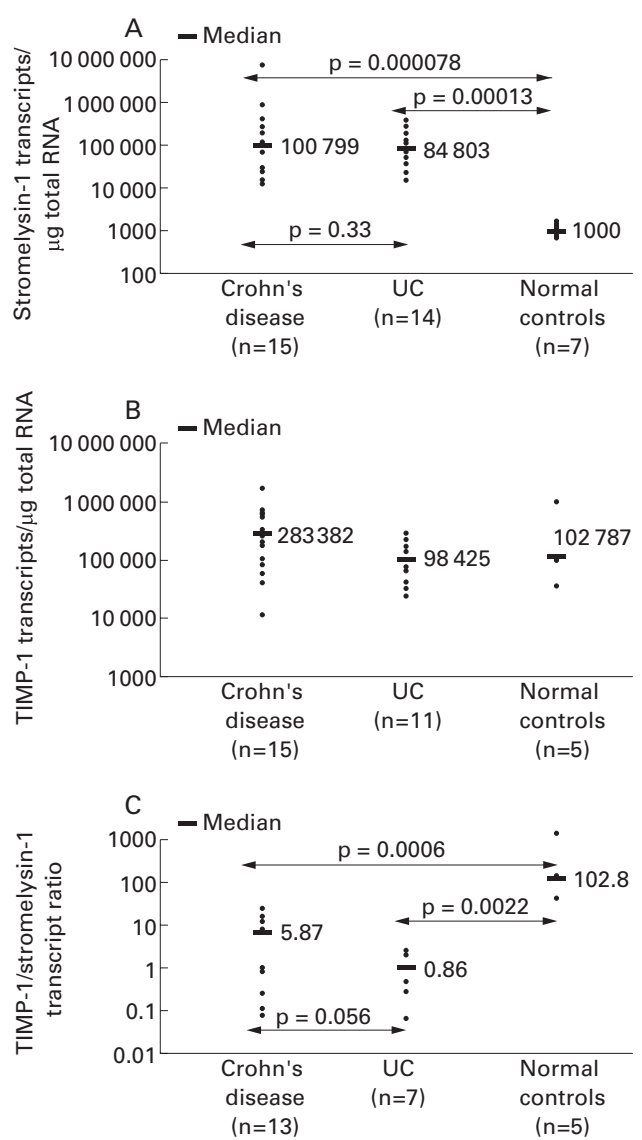

Figure 1 Stromelysin-1 (A), TIMP-1 (B) $m R N A$, and their ratio $(C)$ in active Crohn's disease and ulcerative colitis (UC), and in normal controls, as measured by quantitative reverse transcription-polymerase chain reaction (RT-PCR). difference between normal controls and the IBD patient group (data not shown).

PROTEIN ANALYSIS

Western blot analysis of stromelysin-1 protein showed similar results as RT-PCR. There was a clear increase in stromelysin-1 in the biopsies obtained from children with active IBD compared with normal controls (fig 2A). Not only were the 58 and $60 \mathrm{kDa}$ bands of inactive stromelysin-1 elevated in active IBD, but the lower molecular weight active forms of the enzyme $(56 / 54,44 \mathrm{kDa})$ were also clearly visible, especially in children with UC. Only the inactive enzyme was seen in control patients.

There was no significant difference in TIMP-1 protein levels between the three groups (fig 2B). The ratios of TIMP-1/ stromelysin-1 were significantly different between Crohn's disease and controls $(p<0.0009)$ and UC and controls $(p<0.0003)$. There was no significant difference between Crohn's disease and UC $(p<0.28$, MannWhitney U test) (fig 2C). Figure 3 shows a representative western blot.

Interstitial collagenase and gelatinase $\mathrm{B}$ were also detected by western blotting, but their levels were very low and there were no significant differences between normal and disease tissues (data not shown).
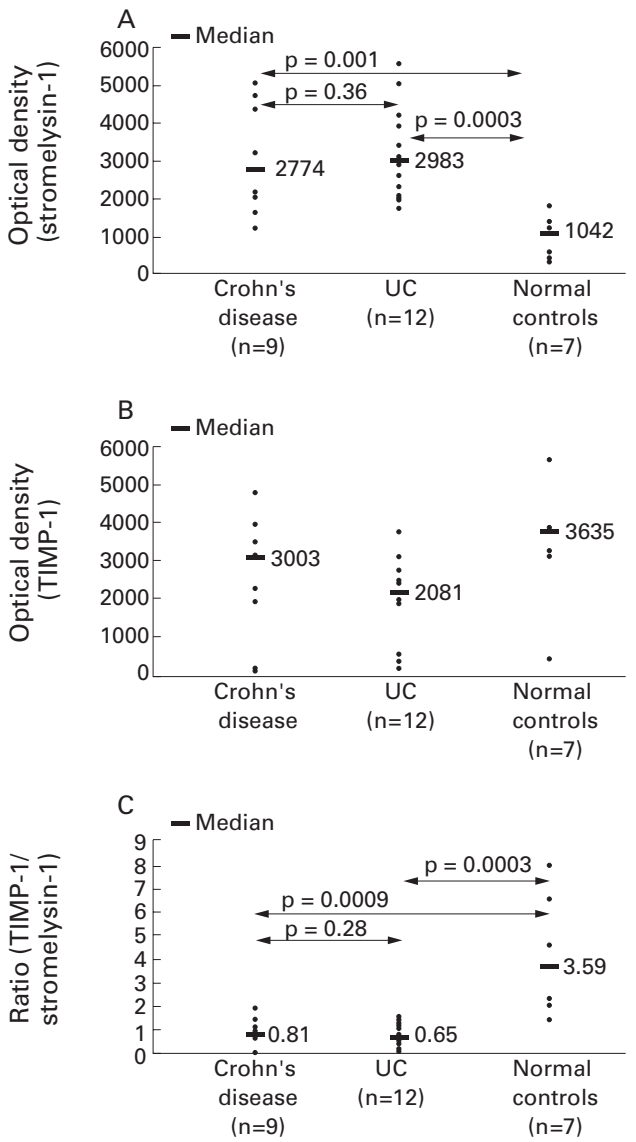

Figure 2 Quantitative analysis of stromelysin-1 protein (A), TIMP-1 protein (B), and their ratio (C) in active Crohn's disease and ulcerative colitis (UC), and in normal controls, as measured by densitometric scanning of western blots. 


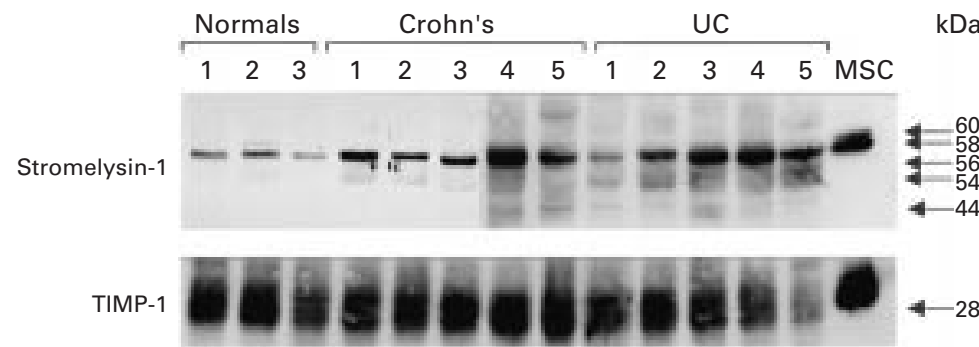

Figure 3 Western blot showing increased expression of stromelysin-1 in children with active Crohn's disease and ulcerative colitis (UC) compared with normal controls (60 and $58 \mathrm{kDa}$ latent bands, with 56, 54, and $44 \mathrm{kDa}$ active enzyme bands).

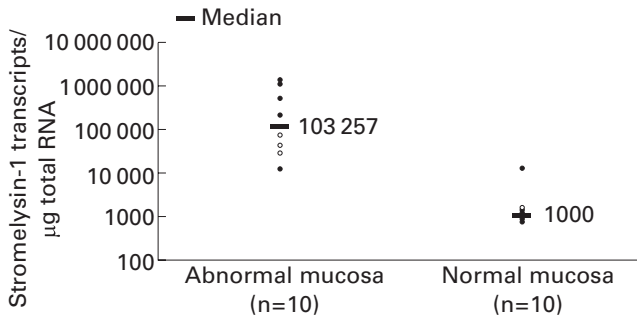

Figure 4 Stromelysin-1 mRNA in endoscopically normal and abnormal large bowel mucosa in children with IBD (Crohn's disease $(\bullet)$, ulcerative colitis $(0)$.

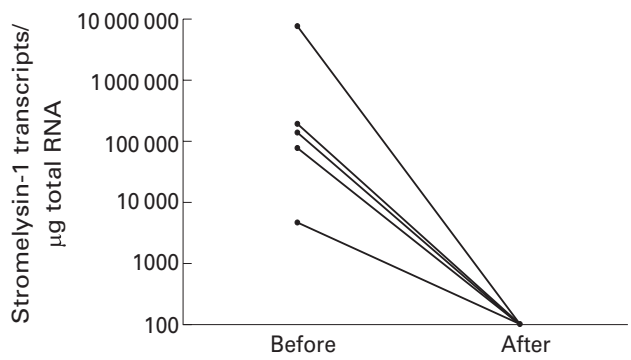

Figure 5 Stromelysin-1 $m R N A$ before and after enteral nutrition in children with Crohn's disease.

STROMELYSIN-1 AND TIMP-1 IN ENDOSCOPICALLY ABNORMAL VERSUS NORMAL MUCOSA

Ten children had paired biopsies obtained from endoscopically "normal" and "abnormal" areas within their large bowel. Stromelysin-1 transcripts was significantly elevated $(\mathrm{p}<0.00008$, Mann-Whitney U test) in all areas of endoscopically abnormal mucosa compared with areas that were endoscopically normal (fig 4). TIMP-1 was not significantly different between these areas.

POST ENTERAL NUTRITION

Following an eight week treatment course of a whole protein, casein based, polymeric diet, there was complete downregulation of stromelysin-1 transcripts to normal values (fig 5) and stromelysin-1 protein production was also decreased (data not shown).

\section{Discussion}

We have demonstrated upregulation of stromelysin- 1 transcripts and its protein in the inflamed gut of children with IBD. The inhibitor TIMP-1 was abundant but unaltered in diseased and normal mucosa, both at the transcript and protein levels. There was 100-fold difference in TIMP-1/stromelysin-1 transcript ratios between normal children and those with active IBD whereas there was a fivefold differ- ence in the ratio of protein band intensities between normal and diseased biopsies. Although these units may not directly reflect the biological activities or quantities of stromelysin-1 and TIMP-1 transcripts and protein, the consistently large differences suggest that the imbalance of this enzyme and its inhibitor plays a significant role in the pathogenesis of IBD. In this study we have also shown the absence of stromelysin-1 transcripts in areas of endoscopically and histologically normal large bowel in children with clinically active IBD. This is the first quantitative evidence showing the absolute number of transcripts of stromelysin-1 and TIMP-1 in gut disease. As matrix degradation and ulceration are major features of IBD, we would suggest that these are mediated by excess stromelysin-1.

This work extends the studies of SaarialhoKere and colleagues ${ }^{13}$ who studied MMP and TIMP-1 transcripts in gastrointestinal ulcers by in situ hybridisation. Stromelysin-1 mRNA containing cells were abundant and increased at the edge of ulcers of patients with IBD, as were TIMP-1 mRNA containing cells. We could not confirm increased TIMP-1 transcripts near ulcers as we deliberately chose not to take a biopsy at the edge of an ulcer. Two previous studies have examined MMP proteins in IBD. ${ }^{14}{ }^{19}$ Bailey and colleagues, ${ }^{14}$ using immunofluorescence staining, showed only a slight increase in immunoreactive stromelysin- 1 in areas of smooth muscle proliferation and of mucosal damage in patients with Crohn's disease. Staining was diffuse and localised to the extracellular space and was probably active enzyme binding to matrix proteins. In this regard it is noteworthy that smaller molecular weight active bands of stromelysin-1 were readily detectable in IBD mucosa by western blotting in this study. Baugh et al showed upregulation of gelatinase $\mathrm{B}$ in IBD. ${ }^{19}$ Both Bailey et al and Baugh et al showed that the inflammatory infiltrate was associated with increased numbers of polymorphonuclear leucocytes that stained positive for gelatinase B in Crohn's disease. For this reason we did not attempt to study gelatinase B in this study.

The cellular source of stromelysin-1 in IBD is almost certainly lamina propria stromal cells. ${ }^{20}$ Stromelysin-1 mRNA positive cells are colocalised with $\alpha$ smooth muscle actin positive cells in a T cell mediated tissue injury model. ${ }^{21}$ Stimulation of mucosal stromal cell lines with IL- $1 \beta$ and TNF- $\alpha$ caused a significant increase in stromelysin-1 production. ${ }^{12}$ It is well established that these two cytokines are elevated in the mucosa of patients with active IBD $^{22}{ }^{23}$ and they are probably also responsible for enhanced stromelysin-1 production.

One of the most striking features of this study was the fact that TIMP-1 transcripts and protein did not increase in inflamed gut mucosa. TNF- $\alpha$ downregulates TIMP-1 more effectively than IL-1 $\beta,{ }^{24}$ but IL-6 also affects TIMP-1 and TIMP-3 expression. ${ }^{25-27}$ IL- 6 is elevated in the mucosa of patients with IBD. Although there is a significant increase in IL-6 in the involved mucosa of patients with UC, in 
$\mathrm{CD}$ there is no difference between involved and non-involved mucosa. ${ }^{28}$ Why IL-6 fails to increase TIMP-1 production remains unclear. An IL-6/IL-6 receptor complex has been found to upregulate TIMP-1 while inhibiting expression of TIMP-3 in human synovial cells. ${ }^{25}$ TIMP-3 is exclusively matrix bound and its inhibition may therefore contribute to local ECM breakdown during inflammation, while a systemic increase in TIMP-1 prevents more widespread ECM damage.

In other inflammatory conditions elevated stromelysin-1 is seen without concomitant TIMP-1 elevation. Stimulation of cultured scleral fibroblasts by TNF- $\alpha$ from patients with necrotising scleritis resulted in a twofold increase in TIMP-1 mRNA compared with a sevenfold increase in stromelysin-1 mRNA. ${ }^{29}$ There was similarly no increase in TIMP-1 mRNA in the aqueous humour of patients with uveitis while MMPs were clearly increased. ${ }^{30}$ In contrast, Yoshihara and colleagues ${ }^{31}$ and others have shown a clear increase in serum TIMP-1 during active rheumatoid arthritis, but importantly, levels within the synovial fluid remained unchanged. In studies of toxic liver damage in humans and rodents, an early increase in TIMP-1 mRNA - that is, during acute inflammation-was noted. ${ }^{32}{ }^{33}$ This suggests that there may be a temporal peak in the inhibitor early in the course of the inflammatory response, although we are unable to document this in well established human IBD. It appears that local concentrations of TIMP-1 and MMPs are differentially regulated in inflammatory conditions in different tissues.

The lack of an increase in TIMP-1 may however be compensated for by an increase in other TIMPs. TIMP-2, TIMP-3, ${ }^{44}$ and TIMP $-4^{35}$ have also been identified in the mucosa of patients with IBD. TIMP-3 is present in both normal and inflamed gut mucosa, yet there are far fewer positive cells within normal tissue compared with both IBD and ischaemic colitis. TIMP-3 is predominantly seen within macrophage- or fibroblastlike cells in the lamina propria and in endothelial cells. ${ }^{36}$ In ongoing studies we are analysing other TIMPs within the mucosa of children with IBD to determine their distribution and relationship to disease activity.

We have also shown that enteral nutrition can return stromelysin-1 transcript levels to normal in active Crohn's disease. However, we would emphasise that this study was only preliminary and not controlled. Indeed, based on the fact that stromelysin is not elevated in biopsies from endoscopically normal mucosa, we would predict that any therapy which reduces mucosal inflammation will lead to a reduction in stromelysin. While the mechanism of action of enteral nutrition is unclear, it has been shown to downregulate mucosal IL-1 $\beta$, IL-2, and IFN- $\gamma^{37}$ and we have shown previously that IL-1 $\beta$ is a potent inducer of stromelysin in gut myofibroblasts. ${ }^{12}$

As cytokines have such a clear effect on MMPs, it may be that downregulation of these by enteral nutrition, steroids, and immunosuppressants causes mucosal healing. Recent atten- tion has focused on the ability of the monoclonal cA2 TNF- $\alpha$ antibody to heal aggressive and fistulising Crohn's disease. ${ }^{38}$ Blockade of TNF- $\alpha$ has a marked effect on the ability of the matrix to restore itself. Brennan and colleagues ${ }^{39}$ clearly showed downregulation of both collagenase-1 and stromelysin- 1 in the serum of patients with active rheumatoid arthritis after treatment with antirheumatoid arthritis factor. In an ex vivo model of $\mathrm{T}$ cell mediated matrix degradation in explants of human fetal intestine, we have also shown that blockade of TNF- $\alpha$ prevents matrix degradation concomitant with inhibition of stromelysin-1 production. ${ }^{40}$ We would suggest that one of the ways in which anti-TNF- $\alpha$ heals fistulae is by blocking TNF- $\alpha$ induced matrix degradation and thus allowing the fistulas to heal.

Finally, we would like to suggest that excess MMPs induced by excess cytokines play a major role in the degradation of the matrix in the mucosa leading to ulceration, and are probably the final step in the cascade of events leading to tissue injury. Agents that inhibit MMPs have been developed by a number of companies and while it would be unwise to use MMP inhibitors as a single therapy for active IBD, we feel that they may provide another target to help heal the mucosa. We thank Dr Simon Murch and Dr Mike Thomson for their
help in obtaining biopsies. We are also grateful to Dr Franck Arnaud-Battandier of Nestlé Clinical Nutrition for the supply of Arnaud-Battandier of Nestle Clinical Nutrition for the supply of Dr G S Schultz, University of Florida (NIH grant EY05587). Dr G S Schultz, University of Florida (NIH grant EY05587).
This project was supported by Crohn's in Childhood Research Association (CICRA) London, Biotechology and Biological Sciences Research Council (BBSRC) UK, and European Union grant ERBFMRXCT9.

1 Woessner JFJ. Matrix metalloproteinases and their inhibitors in connective tissue remodeling. FASEB F 1991;5:2145-54. 2 Mignatti P, Rifkin DB. Plasminogen activators and matrix metalloproteinases in angiogenesis. Enzyme Protein 1996; 49:117-37.

3 Salamonsen LA. Matrix metalloproteinases and endometrial remodelling. Cell Biol Int 1994;18:1139-44.

4 Uria JA, Ferrando AA, Velasco G, et al. Structure and expression in breast tumors of human TIMP-3, a new member of the metalloproteinase inhibitor family. Cancer Res 1994;54:2091-4.

5 Delaisse JM, Eeckhout Y, Sear C, et al. A new synthetic inhibitor of mammalian tissue collagenase inhibits bone resorption in culture. Biochem Biophys Res Commun 1985;133:483-90.

6 Witte MB, Thornton FJ, Kiyama T, et al. Metalloproteinase inhibitors and wound healing: a novel enhancer of wound strength. Surgery 1998;124:464-70.

7 Vaalamo M, Weckroth M, Puolakkainen P, et al. Patterns of matrix metalloproteinase and TIMP-1 expression in chronic and normally healing human cutaneous wounds. Br f Dermatol 1996;135:52-99.

8 Grant GA, Goldberg GI, Wilhelm SM, et al. Activation of extracellular matrix metalloproteases by proteases and organomercurials. Matrix Suppl 1992;1:217-33.

9 Eeckhout Y, Vaes G. Further studies on the activation of procollagenase, the latent precursor of bone collagenase. Effects of lysosomal cathepsin B, plasmin and kallikrein, Effects of lysosomal cathepsin B, plasmin and kallikrein,
and spontaneous activation. Biochem $\mathcal{f} 1977 ; 166: 21-31$.

10 Eeckhout Y, Vaes G. Proceedings: Activation of the inactive precursor of collagenase by kallikrein and plasmin. Arch Int Physiol Biochim 1974;82:7866.

11 Pender SL, Lionetti P, Murch SH, et al. Proteolytic degradation of intestinal mucosal extracellular matrix after lamina propria T cell activation. Gut 1996;39:284-90.

12 Pender SL, Tickle SP, Docherty AJ, et al. A major role for matrix metalloproteinases in $\mathrm{T}$ cell injury in the gut. $\mathcal{F}$ Immunol 1997;158:1582-90.

13 Saarialho-Kere UK, Vaalamo M, Puolakkainen $\mathrm{P}$, et al. Enhanced expression of matrilysin, collagenase, and stromelysin-1 in gastrointestinal ulcers. Am f Pathol 1996; 148:519-26.

14 Bailey CJ, Hembry RM, Alexander A, et al. Distribution of the matrix metalloproteinases stromelysin, gelatinases A and $\mathrm{B}$, and collagenase in Crohn's disease and normal intestine. f Clin Pathol 1994;47:113-16. 
15 Beattie RM, Nicholls SW, Domizio P, et al. Endoscopic assessment of the colonic response to corticosteroids in 373-9.

16 Hyams JS, Ferry GD, Mandel FS, et al. Development and validation of a pediatric Crohn's disease activity index. $f$ Pediatr Gastroenterol Nutr 1991;12:439-47.

17 Monteleone G, MacDonald TT, Wathen NC, et al. Enhancing lamina propria Th1 cell responses with interleukin 12 produces severe tissue injury. Gastroenterology 1999;117: 1069-77.

18 Tarnuzzer RW, Macauley SP, Farmerie WG, et al. Competitive RNA templates for detection and quantitation of growth factors, cytokines, extracellular matrix components and matrix metalloproteinases by RT-PCR. Biotechniques 1996;20:670-4.

19 Baugh MD, Perry MJ, Hollander AP, et al. Matrix metalloproteinase levels are elevated in inflammatory bowel proteinase levels are elevated in inflammatory bowel

20 Gunther U, Matthes H, Herbst H, et al. Phenotype of cells expressing matrix metalloproteinase- 3 in ulcerative colitis. Ann N Y Acad Sci 1998;859:237-40.

21 Pender SL, Breese EJ, Gunther U, et al. Suppression of T cell-mediated injury in human gut by interleukin-10: role of matrix metalloproteinases. Gastroenterology 1998;115 573-83.

22 Murch SH, Braegger CP, Walker-Smith JA, et al. Location of tumour necrosis factor alpha by immunohistochemistry in chronic inflammatory bowel disease. Gut 1993;34:1705-9.

23 Mahida YR, Wu K, Jewell DP. Enhanced production of interleukin 1-beta by mononuclear cells isolated from mucosa with active ulcerative colitis of Crohn's disease. Gut 1989;30:835-8.

24 Yao PM, Maitre B, Delacourt C, et al. Divergent regulation of $92-\mathrm{kDa}$ gelatinase and TIMP-1 by HBECs in response to IL-1beta and TNF-alpha. Am f Physiol 1997;273:L86674 .

25 Gomez DE, Alonso DF, Yoshiji H, et al. Tissue inhibitors of metalloproteinases: structure, regulation and biological metalloproteinases: structure, regulation
functions. Eur $\mathcal{7}$ Cell Biol 1997;74:111-22.

26 Richards CD, Shoyab M, Brown TJ, et al. Selective regulation of metalloproteinase inhibitor (TIMP-1) by oncostatin

$M$ in fibroblasts in culture. F Immunol 1993;150:5596-603.
7 Roeb E, Graeve L, Mullberg J, et al. TIMP-1 protein Roeb E, Graeve L, Mullberg J, et al. TIMP-1 protein
expression is stimulated by IL-1 beta and IL-6 in primary expression is stimulated by IL-1 beta and II
rat hepatocytes. FEBS Lett 1994;349:45-9.

28 Isaacs KL, Sartor RB, Haskill S. Cytokine messenger RNA profiles in inflammatory bowel disease mucosa detected by polymerase chain reaction amplification. Gastroenterology 1992;103:1587-95.

29 Di Girolamo N, Lloyd A, McCluskey P, et al. Increased expression of matrix metalloproteinases in vivo in scleritis tissue and in vitro in cultured human scleral fibroblasts. $A m$ 7 Pathol 1997;150:653-66.

30 Di Girolamo N, Verma MJ, McCluskey PJ, et al. Increased matrix metalloproteinases in the aqueous humor of patients and experimental animals with uveitis. Curr Eye Res 1996; 15:1060-88.

31 Yoshihara Y, Obata K, Fujimoto N, et al. Increased levels of stromelysin-1 and tissue inhibitor of metalloproteinases-1 in sera from patients with rheumatoid arthritis. Arthritis Rheum 1995;38:969-75.

32 Roeb E, Purucker E, Breuer B, et al. TIMP expression in toxic and cholestatic liver injury in rat. $\mathcal{f}$ Hepatol 1997;27:535-44.

33 Muzzillo DA, Imoto M, Fukuda Y, et al. Clinical evaluation of serum tissue inhibitor of metalloproteinases-1 levels in patients with liver diseases. F Gastroenterol Hepatol 1993;8: 437-41.

34 Stetler-Stevenson WG, Krutzsch HC, Liotta LA. Tissue inhibitor of metalloproteinase (TIMP-2). A new member of the metalloproteinase inhibitor family. $f$ Biol Chem 1989;264:17374-88.

35 Leco KJ, Apte SS, Taniguchi GT, et al. Murine tissue inhibitor of metalloproteinases-4 (Timp-4): cDNA isolation and expression in adult mouse tissues. FEBS Lett 1997;401: 213-17.

36 Vaalamo M, Karjalainen-Lindsberg ML, Puolakkainen P, et al. Distinct expression profiles of stromelysin-2 (MMP10), collagenase-3 (MMP-13), macrophage metalloelastase (MMP-12), and tissue inhibitor of metalloproteinases-3 (TIMP-3) in intestinal ulcerations. Am F Pathol 1998;152: 1005-14.

37 Breese EJ, Michie CA, Nicholls SW, et al. The effect of treatment on lymphokine-secreting cells in the intestinal mucosa of children with Crohn's disease. Aliment Pharmacol Ther 1995;9:547-52.

38 Targan SR, Hanauer SB, van Deventer SJ, et al. A short-term study of chimeric monoclonal antibody cA2 to tumor necrosis factor alpha for Crohn's disease. Crohn's Disease cA2 Study Group. N Engl f Med 1997;337:102935.

39 Brennan FM, Browne KA, Green PA, et al. Reduction of serum matrix metalloproteinase 1 and matrix metalloproteinase 3 in rheumatoid arthritis patients following antitumour necrosis factor-alpha (cA2) therapy. Br f Rheumatol 1997;36:643-50.

40 Pender SL, Fell JM, Chamow SM, et al. A p55 TNF receptor immunoadhesin prevents $\mathrm{T}$ cell-mediated intestinal injury by inhibiting matrix metalloproteinase production. $\mathcal{F}$ Immunol 1998;160:4098-103. 